\title{
Fluorescent Anti-MUC5AC Brightly Targets Pancreatic Cancer in a Patient-derived Orthotopic Xenograft
}

\author{
MICHAEL A. TURNER ${ }^{1,2}$, HANNAH M. HOLLANDSWORTH ${ }^{1,2}$, HIROTO NISHINO ${ }^{1,2}$, \\ SIAMAK AMIRFAKHRI ${ }^{1,2}$, THINZAR M. LWIN $^{1}$, ANDREW M. LOWY ${ }^{1}$, \\ SUKHWINDER KAUR ${ }^{3}$, GOPALAKRISHNAN NATARAJAN ${ }^{3}$, KAVITA MALLYA ${ }^{3}$, \\ ROBERT M. HOFFMAN ${ }^{1,2,4}$, SURINDER K. BATRA ${ }^{3}$ and MICHAEL BOUVET ${ }^{1,2}$ \\ ${ }^{1}$ Department of Surgery, University of California San Diego, La Jolla, CA, U.S.A.; \\ ${ }^{2}$ VA San Diego Healthcare System, San Diego, CA, U.S.A.; \\ ${ }^{3}$ Department of Biochemistry and Molecular Biology, University of Nebraska Medical Center, Omaha, NE, U.S.A.; \\ ${ }^{4}$ AntiCancer, Inc., San Diego, CA, U.S.A.
}

\begin{abstract}
Background: Overexpression of mucin-5AC (MUC5AC) makes it a targetable biomarker in pancreatic cancer. The present study evaluated tumor targeting with a MUC5AC antibody conjugated to a near-infrared dye in a patient-derived orthotopic xenograft (PDOX) mouse model. Materials and Methods: MUC5AC monoclonal antibody was conjugated to the near-infrared dye IRDye $800 \mathrm{CW}$ to synthesize MUC5AC-IR800. PDOX models were established by implanting a high-MUC5AC-expressing patient-derived pancreatic tumor on the pancreas of nude mice. After 4 weeks of PDOX tumor growth, mice were imaged after receiving MUC5AC-IR800 (75 $\mu \mathrm{g})$ intravenously. Results: In the PDOX models, MUC5AC-IR800 selectively and brightly targeted the pancreatic tumor (tumor to background ratio: $2.46 \pm 0.465)$. Conclusion: MUC5AC-IR800 provides distinct visualization of pancreatic tumors. MUC5AC-IR800 may be used clinically in the future to improve pancreatic cancer resection. This novel fluorescent probe is also promising for targeting of pre-malignant pancreatic lesions with subsequent resection under fluorescence guidance.
\end{abstract}

Pancreatic cancer is the fourth-leading cause of cancer death in the United States $(1,2)$. Over 60,000 people are estimated to be diagnosed with pancreatic cancer and $>48,000$ will die from it

This article is freely accessible online.

Correspondence to: Michael Bouvet, MD, UCSD Moores Cancer Center, 3855 Health Sciences Drive \#0987, La Jolla, CA 920930987, U.S.A. Tel: +1 8588226191, Fax: +1 8588226192, e-mail: mbouvet@ucsd.edu

Key Words: Pancreatic cancer, mucin, MUC5AC, antibody, tumorspecific imaging, fluorescence guided surgery, PDOX. in the United States in 2021 (2). Of people diagnosed with pancreatic cancer, $90 \%$ of them will die from the disease (1).

The only current curative treatment for pancreatic cancer is surgical resection (1). However, most patients are diagnosed with advanced disease, with only $15-20 \%$ considered surgical candidates (3). Of those that undergo surgical resection, $50-80 \%$ have positive microscopic resection margins (R1), a poor-prognosis indicator. Over $60 \%$ of patients will develop recurrence of disease within 2 years of their operation $(4,5)$. Therefore, improved methods of pancreatic cancer therapeutics are needed.

Mucins, glycoproteins involved in cell signaling and barrier protection, are overexpressed in pancreatic cancer $(6,7)$; specifically, mucin-5AC (MUC5AC), which has minimal or no expression in healthy pancreatic tissue (8-10). Jonckheere et al. performed mucin staining in normal pancreatic tissue, pancreatic intraepithelial neoplasia (PanIN) and pancreatic ductal adenocarcinoma (PDAC). MUC5AC expression was found in $2-4 \%$ of normal ducts but its overexpression was found in 70\% of PanIN IA cases and 85\% of PDAC (9). In the present study, fluorescent-tagged antibodies to MUC5AC were tested with tumor lysates of pancreatic cancer and pancreaticcancer patient-derived orthotopic xenograft (PDOX) models.

\section{Materials and Methods}

Mice. Athymic nude mice, aged 4-6 weeks, were purchased from The Jackson Laboratory (Bay Harbor, ME, USA). The mice were housed in a barrier facility and fed an autoclaved laboratoryapproved diet. The mice were anesthetized with intraperitoneal injection of a solution of xylazine, ketamine and phosphate-buffered saline (PBS) prior to all surgical procedures. At the conclusion of the study, mice were euthanized with $\mathrm{CO}_{2}$ inhalation or cervical dislocation. All studies were approved by the San Diego Veterans Administration Medical Center Institutional Animal Care and Use Committee (animal-use protocol A17-020). 


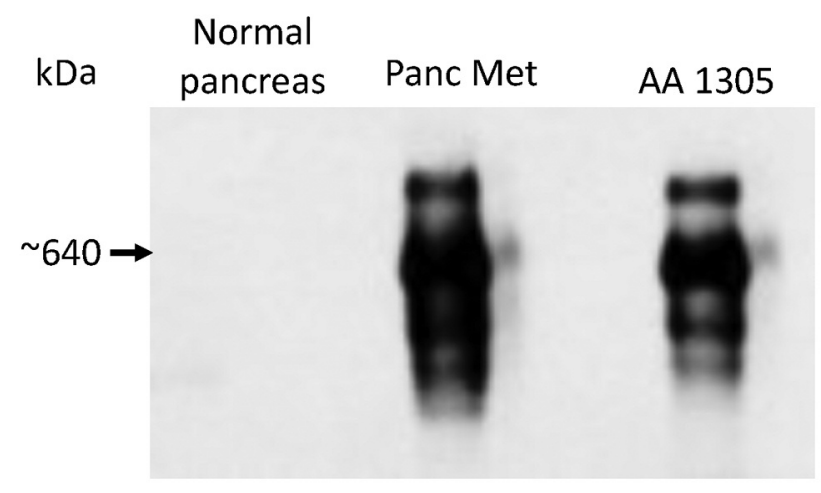

Figure 1. Western blot showing a minimal level of expression of mucin$5 A C$ on the normal pancreas but high levels of expression in a primary patient-derived pancreatic tumor, AA1305, and a patient-derived liver metastasis from a primary pancreatic cancer (PancMet).

Antibody conjugation. Monoclonal MUC5AC antibody (Novus Biologicals, Littleton CO, USA) was conjugated to the near-infrared (NIR) dye IRDye800CW NHS ester (LI-COR Biosciences, Lincoln NE, USA) establishing MUC5AC-IR800. The dye was conjugated to the antibody per the manufacturer's protocol and incubated at room temperature for $2 \mathrm{~h}$ on a shaker plate. After incubation, the antibody-dye conjugate was added to a gel desalting column (Thermo Fisher Scientific, Waltham, MA, USA) to remove excess unbounded dye. The final product was stored at $4^{\circ} \mathrm{C}$.

Western blotting. Tumor lysates were made using a normal human pancreas, a patient-derived pancreatic tumor (AA1305) and a patient-derived liver metastasis (PancMet). Western blotting was performed as previously described (11). Total-protein lysates (80 $\mu \mathrm{g})$ were electrophoretically separated in $2 \%$ sodium dodecyl sulfate-agarose gels prepared using $1.5 \mathrm{M}$ Tris $\mathrm{pH}$ 8.8. Proteins were resolved by horizontal electrophoresis for $4 \mathrm{~h}$ at $100 \mathrm{~V}$ in Trisglycine-sodium dodecyl sulfate buffer. The proteins were transferred onto polyvinylidene-difluoride membranes by overnight horizontal transfer and then blocked in 5\% skim milk in PBS. After blocking, membranes were incubated with primary antibody (CLH2, cat. no. MAB2011; Millipore, Burlington MA, USA) at $4{ }^{\circ} \mathrm{C}$ overnight. Subsequently, membranes were washed with phosphate-buffered saline/tween solution three times and incubated with horseradish peroxidase-labelled anti-mouse secondary antibody for 1 hour at room temperature. After secondary-antibody incubation, membranes were washed three times with phosphate-buffered saline/tween solution. Protein bands were visualized using a chemiluminescence reagent (Luminol enhancer solution and peroxide solution; Thermo Fisher Scientific).

PDOX establishment. A patient-derived primary pancreatic cancer (AA1305) was used in the present study. To establish the cancer subcutaneously in nude mice, tumor fragments $\left(\sim 1 \mathrm{~mm}^{3}\right)$ made from a surgical specimen were implanted in the bilateral flanks and shoulders of the mice $(n=6)$. Tumor fragments were allowed to grow for $\sim 4$ weeks. Next, subcutaneous-grown tumors were harvested, and tumor fragments $\left(\sim 1 \mathrm{~mm}^{3}\right)$ were implanted in the pancreas of the mice $(n=6)$ to create a PDOX model. This was accomplished

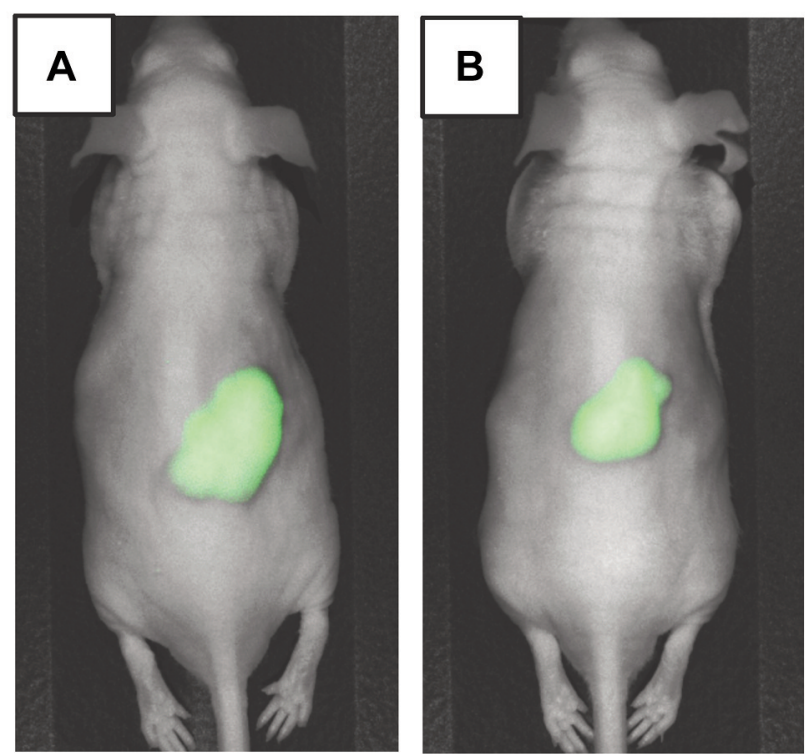

Figure 2. Imaging of subcutaneous models $72 \mathrm{~h}$ after injection with 50 $\mu \mathrm{g}(\mathrm{A})$ and $75 \mu \mathrm{g}(\mathrm{B})$ mucin-5AC monoclonal antibody conjugated to the near-infrared dye IRDye $800 \mathrm{CW}$.

first by anesthetizing the mice then sterilizing their abdomen with a $70 \%$ ethanol solution. A small incision was made in the upper abdomen just left of midline. The mouse's spleen was carefully exposed and retracted cephalad, exposing the pancreas. The tumor fragment was sutured to the body of the pancreas using 8-0 nylon suture (Ethicon Inc., Somerville, NJ, USA) (12). The mouse organs were returned to the peritoneal cavity and the incision was closed with 6-0 nylon suture (Ethicon Inc.). Post-procedural pain was treated with subcutaneous buprenorphine $(25 \mu \mathrm{l})$ reconstituted in PBS. The tumors were allowed to grow for 4 weeks. Male and female mice were used. Patient tumors and normal tissue were obtained with informed consent under UCSD Institutional Review Board approval.

Antibody-dye conjugate dosing and imaging. The antibody-dye conjugate was administered via tail vein injection. In vivo imaging with NIR wavelength $(800 \mathrm{~nm})$ was performed daily in subcutaneous models for $72 \mathrm{~h}$ using a Pearl Trilogy Small Animal Imaging System (LI-COR Biosciences). The Pearl Trilogy Small Animal Imaging System was used to quantify the strength of the NIR signal from the tumor and from the skin which was used as background. A pilot experiment used MUC5AC-IR800 doses of 25, 50 and $75 \mu \mathrm{g}$. After the subcutaneous tumors grew to $1 \mathrm{~cm}^{3}$, mice were divided into three groups to receive 25,50 or $75 \mu \mathrm{g}$ of the antibody-dye conjugate. There were two mice in each group for a total of six subcutaneous models. The mice were imaged at 24,48 and $72 \mathrm{~h}$ after receiving antiMUC5AC. The tumor to background ratio (TBR) was calculated by dividing the peak intensity of the signal from the tumor by the peak intensity of the signal from the skin.

For the PDOX model, mice $(\mathrm{n}=6)$ received MUC5AC-IR800 via tail injection $(75 \mu \mathrm{g})$. An incision was made in the left upperabdominal quadrant of the PDOX models and the pancreas and 

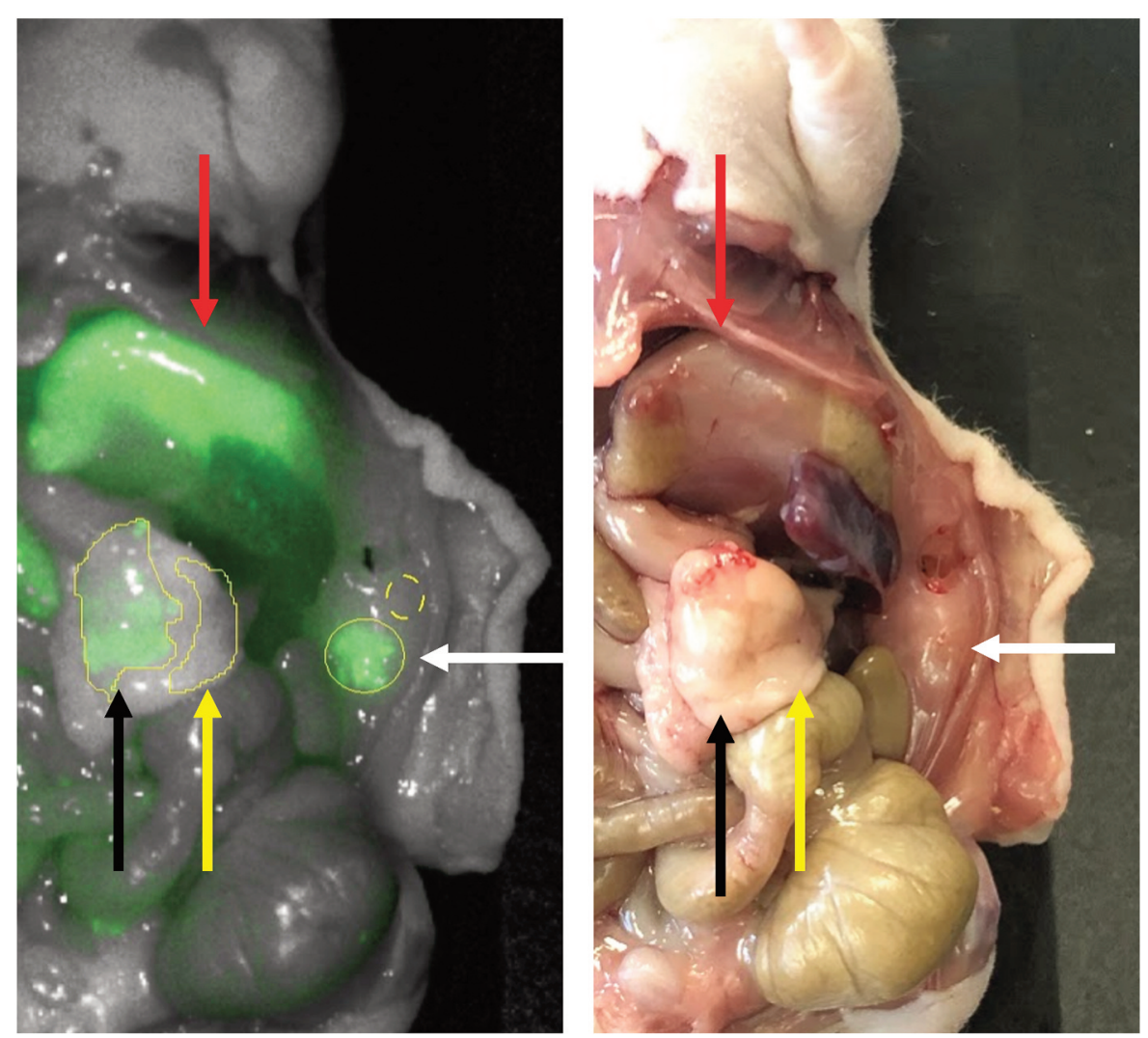

Figure 3. Patient-derived orthotopic xenograft (PDOX) model of AA1305 $72 \mathrm{~h}$ after injection of $75 \mu \mathrm{g}$ of mucin 5AC (MUC5-AC) monoclonal antibody conjugated to the near-infrared dye IRDye800CW. The implanted pancreatic tumor (black arrow) brightly fluoresced compared to normal pancreas surrounding it (yellow arrow). There is also a fluorescence signal from local tumor invasion on the abdominal wall (white arrow) where the peritoneum overlaid the pancreas, as well as fluorescence on the mouse's stomach (red arrow). The fluorescence on the stomach does not represent metastatic spread but normal MUC5AC expression, possibly also fluorescence of the mouse chow.

tumor were carefully exposed. In vivo imaging was performed at 48 hours with the Pearl Trilogy Small Animal Imaging System. At 72 hours, the mice were euthanized and a laparotomy incision was made, exposing the peritoneal cavity and abdominal contents. Imaging with the Pearl Trilogy Small Animal Imaging System was again performed. Normal pancreas was used to calculate the background tissue signal. The TBR was a calculated by dividing the peak tumor signal by the peak background signal.

\section{Results}

MUC5AC expression. Western blotting demonstrated minimal to no MUC5AC expression in normal pancreas obtained from the patient. However, there was a very high level of MUC5AC in the patient-derived pancreatic cancer, AA1305 (Figure 1).

TBR in subcutaneous models. Figure 2 shows imaging at $72 \mathrm{~h}$ of mice which received $50 \mu \mathrm{g}$ and $75 \mu \mathrm{g}$ of MUC5AC-IR800, respectively. The TBRs (average $\pm \mathrm{SD}$ ) at 24,48 and $72 \mathrm{~h}$ for the $25 \mu \mathrm{g}$ group were $1.25 \pm 0.339,1.67 \pm 0.679$ and $1.84 \pm 0.700$, respectively. For the $50 \mu \mathrm{g}$ group, they were $2.44 \pm 1.209$, $3.42 \pm 1.739$ and $3.83 \pm 1.874$, respectively. For the $75 \mu \mathrm{g}$ group, the TBRs were $2.19 \pm 0.085,2.82 \pm 0.177$ and $4.35 \pm 0.177$, respective. The highest TBR was measured at $72 \mathrm{~h}$ in the 75 $\mu$ g-treated group and these conditions were used for the PDOX models.

TBR in the PDOX models. The average TBR at $48 \mathrm{~h}$ was $2.46 \pm 0.465$ and that at $72 \mathrm{~h}$ was $2.183 \pm 0.619$ (Figure 3 ).

No toxicity was observed in the subcutaneous or orthotopic models.

\section{Discussion}

The need for R0 resection is paramount as surgical resection is the only current curative treatment for pancreatic cancer $(4,13)$. Intraoperatively, the surgeon is dependent on visual and tactile cues of the unlabeled tumor to determine appropriate extent of 
resection (13). The need for improvement is shown by the high rates of $\mathrm{R} 1$ resection, reportedly as high as $50-80 \%(4,13)$. There is controversy regarding the wide range of $\mathrm{R} 1$ rates $(<20 \%$ to $>80 \%$ ) reported in the literature (4). This is due to the lack of consensus in defining R1 (although it is accepted that the true $\mathrm{R} 1$ rate is under-reported given the high rate of recurrence in R0 resections) $(4,5,14)$. In the UK and Germany, the superiormesenteric-vein groove is routinely evaluated and considered a margin while in the United States, the superior-mesenteric-vein groove may be evaluated but only the portion facing the superior mesenteric-artery requires mandatory evaluation (14). Moreover, the International Union Against Cancer classification defines R1 as microscopic tumor at specimen edge ( $0 \mathrm{~mm}$ margin), whereas more recent protocols such as guidelines from the British Royal College of Pathologists define R1 as tumor within $1 \mathrm{~mm}$ of the specimen edge $(15,16)$.

Specific fluorescence tumor labeling should improve visualization of primary and metastatic disease wherever it is located (including the uncinate or the superior-mesentericartery groove), enabling more aggressive resection if indicated and safe. Improved tumor margin visualization could lead to an improved rate of $\mathrm{R} 0$ resection and improved disease-free and overall survival. This is the aim of fluorescence-guided surgery (FGS) in oncology.

In the present study, we demonstrated the ability of fluorescent MUC5AC antibodies to selectively target pancreatic cancer tissue compared to normal pancreatic tissue. Westernblot analysis of patient-derived tumor samples demonstrated high expression of MUC5AC, consistent with previouslypublished data regarding mucins and pancreatic cancer $(6,8$, 10). Kim et al. reviewed 68 cases of PDAC. They found MUC5AC staining in the majority of PanIN 1A (71\%), PanIN 1B (89\%), PanIN 2 (88\%), and PanIN 3 (90\%) cases (17). MUC5AC staining was also demonstrated in well-differentiated (100\%), moderately-differentiated (96\%) and poorlydifferentiated (59\%) PDAC specimens (17). This was confirmed by high TBRs in both our subcutaneous and orthotopic models. The different TBRs between the models is expected given the difference between skin and pancreatic tissue. The subcutaneous models are useful for dose-ranging and timing of imaging; however, the orthotopic models are considered more clinically relevant (18). In the orthotopic model, the TBR decrease between 48 and $72 \mathrm{~h}$ is due to the signal of the tumor being stable from 48 to $72 \mathrm{~h}$ but the background tissue (normal pancreas) having a slight increase in signal strength.

A concern with using an antibody to MUC5AC is its binding to other locations expressing MUC5AC ('off-target binding'). MUC5AC is a secretory mucin mainly found on the apical surface of epithelial cells in the respiratory and gastrointestinal. tract (6). MUC5AC expression has been reported in the tracheobronchial lining, gallbladder and the lumen of the lacrimal ducts (6). These sites may have off-target binding and their potential interference with tumor imaging using
MUC5AC fluorescence antibody must be considered. However, in the present study, there was no off-target labeling which interfered with the pancreatic-cancer fluorescence signal.

Since the early 1990s, fluorescence-tagged antibodies have been used to image tumors (13). Our laboratory pioneered FGS of pancreatic cancer in PDOX models using fluorescent antibodies to selectively visualize the tumor, resulting in improved R0 resections, reduced recurrence and increased survival of the mouse models compared to bright light surgery (BLS) (19-21). McElroy et al. demonstrated strong pancreaticcancer-cell binding by a fluorescent antibody probe with minimal background peritumoral-stroma staining (22). Maawy et al. compared carcinoembryonic antigen antibodies conjugated to different dyes of varying wavelengths, showing longer wavelengths were associated with higher TBRs and greater tissue penetration (23). Kaushal et al. demonstrated improved visualization of pancreatic-cancer cells in vitro and in subcutaneous pancreatic cancer models in vivo with fluorescent antibodies to carcinoembryonic antigen (24). Metildi et al. compared FGS to BLS in orthotopic mouse models of pancreatic cancer and showed improved resection rates, diseasefree and overall survival in the FGS arm $(19,20)$. Hiroshima et al. randomized pancreatic PDOX models to neoadjuvant chemotherapy (NAC) with gemcitabine with FGS, FGS alone, BLS with NAC or BLS alone, to determine if FGS in combination with NAC would lead to better outcomes. Their results showed the FGS-plus-NAC arm had reduced rates of metastatic recurrence (25). Furthermore, our laboratory is the pioneer of using fluorescent proteins for in vivo imaging (18, $26,27)$. We previously showed fluorescent protein-labeled tumors are powerful tools for FGS $(28,29)$ and FGS can be used for resection of recurrent tumors expressing the fluorescent protein, which serves as a genetic reporter (30).

Limitations of the present study include the use of immunocompromised mice in the establishment of the PDOX models and the lack of murine pancreas evaluation in the western blot. Another limitation of the PDOX model is the pancreatic tumor is sutured onto the pancreas rather than growing from within it. Another model which may represent a pancreatic tumor growing in the pancreas is the use of patient-derived explants/spheroids/organoids in vitro. Our laboratory has pioneered 3-D sponge-gel histoculture of patient tumors, including pancreatic cancer (31). In future experiments, pancreatic organoids will be labeled with MUC5AC fluorescent antibodies (32). We also did not perform immunohistochemistry with the AA1305 tumors. We have previously studied immunohistochemical staining of individual cells with a labeled antibody to cancer-antigen 199 in an orthotopic model of pancreatic cancer (22). We will perform a similar experiment with the MUC5AC antibody and pancreatic cancer in future studies. Another limitation is the lack of control animals. McElroy et al. used control mice with unlabeled tumors with no fluorescent-tagged antibody 
which demonstrated no fluorescence signal, indicating the signal from the fluorescent antibody-labeled tumor was not from background tissue (22). Hollandsworth et al. used control animals receiving NIR dye alone (not conjugated to an antibody) in a colon-cancer orthotopic model experiment. The control mice demonstrated non-specific fluorescence without localization to the tumor, indicating the fluorescenttagged protein signal was specific $(33,34)$.

The verification of a new antibody to target pancreatic cancer is an important development of the present study. Next steps in developing fluorescent MUC5AC antibodies targeting pancreatic cancer in the PDOX model include determination of the minimal TBR associated with an increased rate of $\mathrm{R} 0$ resection with FGS.

\section{Conclusion}

The anti-MUC5AC fluorescent-tagged antibody successfully targeted pancreatic tumors in subcutaneous and PDOX mouse models. The fluorescent anti-MUC5AC antibody will be used to confirm the principle that FGS can aid in intraoperative decisions for complete resection of pancreatic cancer with minimal disruption of normal tissue.

\section{Conflicts of Interest}

AntiCancer Inc uses PDOX models for contract research. Robert M. Hoffman is a non-salaried associate of AntiCancer Inc. All other Authors have no disclosures.

\section{Authors' Contributions}

Michael A. Turner wrote the manuscript and was actively involved in each step of the experimental process. Hannah Hollandsworth, Siamak Amirfakhri, and Thinzar Lwin were actively involved in the experimental process and contributed to the Materials and Methods section of the article. Surinder K. Batra, Sukhwinder Kaur, Gopalakrishnan Natarajan, and Kavita Mallya contributed antibodies for the experiments and performed western blotting. Robert M. Hoffman revised the manuscript. Michael Bouvet was involved in oversight of the research design, implementation and editing of the article.

\section{Acknowledgements}

This study was funded by VA Merit Review grant numbers 1 I01 BX003856-01A1 and 1 I01 BX004494-01 (MB) and NIH/NCI T32CA121938 (MAT).

\section{References}

1 Ryan DP, Hong TS and Bardeesy N: Pancreatic adenocarcinoma. N Engl J Med 371(22): 2140-2141, 2014. PMID: 25427123. DOI: $10.1056 / \mathrm{NEJMc} 1412266$

2 Siegel RL, Miller KD, Fuchs HE and Jemal A: Cancer statistics, 2021. CA Cancer J Clin 71(1): 7-33, 2021. PMID: 33433946. DOI: $10.3322 /$ caac. 21654
3 Katz MH, Savides TJ, Moossa AR and Bouvet M: An evidencebased approach to the diagnosis and staging of pancreatic cancer. Pancreatology 5(6): 576-590, 2005. PMID: 16110256. DOI: $10.1159 / 000087500$

4 Rau BM, Moritz K, Schuschan S, Alsfasser G, Prall F and Klar E: R1 resection in pancreatic cancer has significant impact on long-term outcome in standardized pathology modified for routine use. Surgery 152(3 Suppl 1): S103-S111, 2012. PMID: 22766366. DOI: 10.1016/j.surg.2012.05.015

5 Van den Broeck A, Sergeant G, Ectors N, Van Steenbergen W, Aerts R and Topal B: Patterns of recurrence after curative resection of pancreatic ductal adenocarcinoma. Eur J Surg Oncol 35(6): 600604, 2009. PMID: 19131205. DOI: 10.1016/j.ejso.2008.12.006

6 Kaur S, Kumar S, Momi N, Sasson AR and Batra SK: Mucins in pancreatic cancer and its microenvironment. Nat Rev Gastroenterol Hepatol 10(10): 607-620, 2013. PMID: 23856888. DOI: $10.1038 /$ nrgastro.2013.120

7 Moniaux N, Andrianifahanana M, Brand RE and Batra SK: Multiple roles of mucins in pancreatic cancer, a lethal and challenging malignancy. Br J Cancer 91(9): 1633-1638, 2004. PMID: 15494719. DOI: 10.1038/sj.bjc.6602163

8 Balagué C, Gambús G, Carrato C, Porchet N, Aubert JP, Kim YS and Real FX: Altered expression of MUC2, MUC4, and MUC5 mucin genes in pancreas tissues and cancer cell lines. Gastroenterology 106(4): 1054-1061, 1994. PMID: 8143972. DOI: $10.1016 / 0016-5085(94) 90767-6$

9 Jonckheere N, Skrypek N and Van Seuningen I: Mucins and pancreatic cancer. Cancers (Basel) 2(4): 1794-1812, 2010. PMID: 24281201. DOI: 10.3390/cancers2041794

10 Kaur S, Smith LM, Patel A, Menning M, Watley DC, Malik SS, Krishn SR, Mallya K, Aithal A, Sasson AR, Johansson SL, Jain M, Singh S, Guha S, Are C, Raimondo M, Hollingsworth MA, Brand RE and Batra SK: A Combination of MUC5AC and CA19-9 improves the diagnosis of pancreatic cancer: a multicenter study. Am J Gastroenterol 112(1): 172-183, 2017. PMID: 27845339. DOI: 10.1038/ajg.2016.482

11 Rachagani S, Torres MP, Kumar S, Haridas D, Baine M, Macha MA, Kaur S, Ponnusamy MP, Dey P, Seshacharyulu P, Johansson SL, Jain M, Wagner KU and Batra SK: Mucin (Muc) expression during pancreatic cancer progression in spontaneous mouse model: potential implications for diagnosis and therapy. J Hematol Oncol 5: 68, 2012. PMID: 23102107. DOI: 10.1186/1756-8722-5-68

$12 \mathrm{Fu} \mathrm{X}$, Guadagni F and Hoffman RM: A metastatic nude-mouse model of human pancreatic cancer constructed orthotopically with histologically intact patient specimens. Proc Natl Acad Sci USA 89(12): 5645-5649, 1992. PMID: 1608975. DOI: 10.1073/ pnas.89.12.5645

13 Lwin TM, Hoffman RM and Bouvet M: The development of fluorescence guided surgery for pancreatic cancer: from bench to clinic. Expert Rev Anticancer Ther 18(7): 651-662, 2018. PMID: 29768067. DOI: 10.1080/14737140.2018.1477593

14 Häberle L and Esposito I: Circumferential resection margin (CRM) in pancreatic cancer. Surg Pract Sci 1: 100006, 2020. DOI: $10.1016 /$ j.sipas.2020.100006

15 Sobin LH, Gospodarowicz MK, and Christian Wittenkind: TNM Classification of Malignant Tumors. New Jersey, John Wiley and Sons, 2011.

16 Campbell F, Cairns A, Duthie F and Feakins R: Dataset for histopathological reporting of carcinomas of the pancreas, ampulla of Vater and common bile duct. Available at: https:// 
www.rcpath.org/uploads/assets/34910231-c106-4629-a2de9e9 ae6f87ac1/G091-Dataset-for-histopathological-reporting-ofcarcinomas-of-the-pancreas-ampulla-of-Vater-and-common-bileduct.pdf [Last accessed on 21st October 2021]

17 Kim GE, Bae HI, Park HU, Kuan SF, Crawley SC, Ho JJ and Kim YS: Aberrant expression of MUC5AC and MUC6 gastric mucins and sialyl Tn antigen in intraepithelial neoplasms of the pancreas. Gastroenterology 123(4): 1052-1060, 2002. PMID: 12360467. DOI: 10.1053 /gast.2002.36018

18 Hoffman RM: Patient-derived orthotopic xenografts: better mimic of metastasis than subcutaneous xenografts. Nat Rev Cancer 15(8): 451-452, 2015. PMID: 26422835. DOI: $10.1038 / \mathrm{nrc} 3972$

19 Metildi CA, Kaushal S, Hardamon CR, Snyder CS, Pu M, Messer KS, Talamini MA, Hoffman RM and Bouvet M: Fluorescence-guided surgery allows for more complete resection of pancreatic cancer, resulting in longer disease-free survival compared with standard surgery in orthotopic mouse models. J Am Coll Surg 215(1): 126-35; discussion 135-6, 2012. PMID: 22632917. DOI: 10.1016/j.jamcollsurg.2012.02.021

20 Metildi CA, Kaushal S, Pu M, Messer KA, Luiken GA, Moossa AR, Hoffman RM and Bouvet M: Fluorescence-guided surgery with a fluorophore-conjugated antibody to carcinoembryonic antigen (CEA), that highlights the tumor, improves surgical resection and increases survival in orthotopic mouse models of human pancreatic cancer. Ann Surg Oncol 21(4): 1405-1411, 2014. PMID: 24499827. DOI: 10.1245/s10434-014-3495-y

21 Tran Cao HS, Kaushal S, Metildi CA, Menen RS, Lee C, Snyder CS, Messer K, Pu M, Luiken GA, Talamini MA, Hoffman RM and Bouvet M: Tumor-specific fluorescence antibody imaging enables accurate staging laparoscopy in an orthotopic model of pancreatic cancer. Hepatogastroenterology 59(118): 1994-1999, 2012. PMID: 22369743. DOI: 10.5754/hge11836

22 McElroy M, Kaushal S, Luiken GA, Talamini MA, Moossa AR, Hoffman RM and Bouvet M: Imaging of primary and metastatic pancreatic cancer using a fluorophore-conjugated anti-CA19-9 antibody for surgical navigation. World J Surg 32(6): 1057-1066, 2008. PMID: 18264829. DOI: 10.1007/s00268-007-9452-1

23 Maawy AA, Hiroshima Y, Kaushal S, Luiken GA, Hoffman RM and Bouvet M: Comparison of a chimeric anti-carcinoembryonic antigen antibody conjugated with visible or near-infrared fluorescent dyes for imaging pancreatic cancer in orthotopic nude mouse models. J Biomed Opt 18(12): 126016, 2013. PMID: 24356647. DOI: 10.1117/1.JBO.18.12.126016

24 Kaushal S, McElroy MK, Luiken GA, Talamini MA, Moossa AR, Hoffman RM and Bouvet M: Fluorophore-conjugated antiCEA antibody for the intraoperative imaging of pancreatic and colorectal cancer. J Gastrointest Surg 12(11): 1938-1950, 2008. PMID: 18665430. DOI: 10.1007/s11605-008-0581-0

25 Hiroshima Y, Maawy A, Zhang Y, Murakami T, Momiyama M, Mori R, Matsuyama R, Katz MH, Fleming JB, Chishima T, Tanaka K, Ichikawa Y, Endo I, Hoffman RM and Bouvet M: Metastatic recurrence in a pancreatic cancer patient derived orthotopic xenograft (PDOX) nude mouse model is inhibited by neoadjuvant chemotherapy in combination with fluorescenceguided surgery with an anti-CA 19-9-conjugated fluorophore. PLoS One 9(12): e114310, 2014. PMID: 25463150. DOI: 10.1371 /journal.pone .0114310
26 Yang M, Baranov E, Jiang P, Sun FX, Li XM, Li L, Hasegawa S, Bouvet M, Al-Tuwaijri M, Chishima T, Shimada H, Moossa AR, Penman S and Hoffman RM: Whole-body optical imaging of green fluorescent protein-expressing tumors and metastases. Proc Natl Acad Sci USA 97(3): 1206-1211, 2000. PMID: 10655509. DOI: 10.1073/pnas.97.3.1206

27 Chishima T, Miyagi Y, Wang X, Yamaoka H, Shimada H, Moossa $\mathrm{AR}$ and Hoffman RM: Cancer invasion and micrometastasis visualized in live tissue by green fluorescent protein expression. Cancer Res 57(10): 2042-2047, 1997. PMID: 9158003.

28 Kishimoto H, Zhao M, Hayashi K, Urata Y, Tanaka N, Fujiwara $\mathrm{T}$, Penman $\mathrm{S}$ and Hoffman RM: In vivo internal tumor illumination by telomerase-dependent adenoviral GFP for precise surgical navigation. Proc Natl Acad Sci USA 106(34): 1451414517, 2009. PMID: 19706537. DOI: 10.1073/pnas.0906388106

29 Metildi CA, Kaushal S, Snyder CS, Hoffman RM and Bouvet M: Fluorescence-guided surgery of human colon cancer increases complete resection resulting in cures in an orthotopic nude mouse model. J Surg Res 179(1): 87-93, 2013. PMID: 23079571. DOI: $10.1016 /$ j.jss.2012.08.052

30 Kishimoto H, Aki R, Urata Y, Bouvet M, Momiyama M, Tanaka N, Fujiwara T and Hoffman RM: Tumor-selective, adenoviralmediated GFP genetic labeling of human cancer in the live mouse reports future recurrence after resection. Cell Cycle 10(16): 27372741, 2011. PMID: 21785265. DOI: 10.4161/cc.10.16.16756

31 Hoffman RM and Freeman AE: In vivo-like growth patterns of multiple types of tumors in Gelfoam ${ }^{\circledR}$ histoculture. Methods Mol Biol 1760: 19-28, 2018. PMID: 29572790. DOI: 10.1007/9781-4939-7745-1_3

32 Driehuis E, Gracanin A, Vries RGJ, Clevers H and Boj SF: Establishment of pancreatic organoids from normal tissue and tumors. STAR Protoc 1(3): 100192, 2020. PMID: 33377086. DOI: $10.1016 /$ j.xpro.2020.100192

33 Hollandsworth HM, Amirfakhri S, Filemoni F, Schmitt V, Wennemuth G, Schmidt A, Hoffman RM, Singer BB and Bouvet M: Anti-carcinoembryonic antigen-related cell adhesion molecule antibody for fluorescence visualization of primary colon cancer and metastases in patient-derived orthotopic xenograft mouse models. Oncotarget 11(4): 429-439, 2020. PMID: 32064046. DOI: 10.18632/oncotarget.27446

34 Hollandsworth HM, Amirfakhri S, Filemoni F, Hoffman RM, Molnar J, Yazaki PJ and Bouvet M: Humanized anti-tumorassociated glycoprotein-72 for submillimeter near-infrared detection of colon cancer in metastatic mouse models. J Surg Res 252: 1621, 2020. PMID: 32217350. DOI: 10.1016/j.jss.2020.02.017 